\section{GEODÉSICOS POST TERREMOTO. INVESTIGACIÓN APLICADA EN LA EMERGENCIA}

Pedro Serrano Rodríguez ${ }^{1}$

\section{Resumen}

Tres días después del terremoto del 27 de febrero de 2010 en la zona central de Chile, la Unidad de Arquitectura Extrema, ARQ-X, del Departamento de Arquitectura de la Universidad Técnica Federico Santa María (UTFSM), propuso un programa de acción rápido que fuese capaz, en el corto plazo de la post emergencia, de desarrollar dentro de su marco de investigación en geodésicos un sistema productivo y de montaje de coberturas geodésicas muy baratas, que permitiesen cubrir rápidamente enseres, reuniones y actividades escolares en los pueblos más afectados.

La investigación departamental con estructuras geodésicas en diversos materiales y diferentes métodos constructivos venía desde el año 2000. Se te-

\section{POST-EARTHOUAKE GEODESICS. APPLIED RESEARCH ON EMERGENCIES}

Pedro Serrano Rodríguez ${ }^{1}$

\section{Abstract}

Three days after the February 27, 2010 earthquake that struck central Chile, the Extreme Architecture Unit, ARQ-X, under the Architecture Department at Technical University Federico Santa María, proposed a rapid action plan capable of developing in the short term a low cost productive and assemblage geodetic coverage system that would allow to quickly to coordinate equipment, meetings and school activities in the most affected areas.

The department-based research on geodetic structures application in different materials and building methods dates from 2000. Within a week, considering the existing knowledge 
nía el conocimiento y la experiencia y fue posible, en una semana, desarrollar el taller productivo con voluntarios estudiantes, gestionar fondos rápidos con la Universidad y con aportes extranjeros y lograr un sistema productivo eficiente que llegó con las primeras coberturas a terreno antes de fines de marzo 2010.

"Esto no es una vivienda" era la primera advertencia que tenían impresas las coberturas geodésicas, que, sobre todo, no fueron regalos a la gente sino que sistemas desplegables, trasladables y almacenables para otras emergencias.

Este escrito desarrolla el esquema estructural, el proceso productivo, la difusión web y los resultados obtenidos por la iniciativa.

PALABRAS CLAVE: TERREMOTO; POST EMERGENCIA; COBIJO TRANSITORIO; GEODÉSICAS.

Fecha de recepción: 07.03.11

Fecha de aceptación: 10.06.11

1 Chile. Ingeniero Electrónico de la Universidad Técnica Federico Santa María. Director de la Unidad de Arquitectura extrema AR0-X UTFSM, Presidente de la Fundación Terram para el desarrollo sustentable. Académico del Departamento de Arquitectura, Universidad Técnica Federico Santa María. Email: pedro. serrano@usm.cl and experience it was possible to carry out a productive workshop with volunteer students, quickly raise funds from the University and foreign collaborators, and develop an efficient productive system that by the end of March, 2010 was working in the affected areas.

"This is not housing" was the first warning printed on the geodetic coverage; these structures were not gifts to people, but foldable, movable and storable systems intended to be used in future emergencies.

This paper describes the structural scheme, productive process, web distribution, and results achieved by this initiative.

KEY WORDS: EARTHOUAKE, POST-EMERGENCY, TRANSITORY SHELTER, GEODESICS

1 Chile. Electronic engineer, Federico Santa María Technical University. Director, Extreme Arquitecture Unit ARO-X USM, President of Terram Foundation for sustainable development. Academic, Faculty of Architecture, Federico Santa María Technical University. email: pedro.serrano@usm.cl 


\section{Introducción}

\section{MARCO DE REFERENCIA}

Las catástrofes tienen varias etapas, también descritas en la literatura como fases, que se reconocen en su tratamiento y en las formas de reacción por parte de las instituciones y la gente. Según Cesar San Juan², en su libro Catástrofes y ayuda de Emergencia, muchas de estas fases están asociadas a los aspectos físicos y otras a los aspectos psicológicos y psicosociales devenidos de su ocurrencia.

En la catástrofe chilena del terremoto del 27 de Febrero de 2010, las fases físicas reconocidas son:

- La fase cero, que es el origen, la catástrofe misma que puede durar, como en el caso del terremoto de 2010 en Chile, solo 3 minutos, según el informe oficial de geofísica de la U. de Chile, y 20 minutos posteriores para la llegada de la primera ola del tsunami. ${ }^{3}$

- Para algunos autores hay una fase que podríamos llamar aquí "cero menos uno", que es prevenir, sobre lo cual tenemos interesante bibliografía nacional ${ }^{4}$.

SAN JUAN GUILLÉN, César, 2001

UNIVERSIDAD DE CHILE, Departamento de Geofísica, Servicio Sismológico. 2010

4 AHUMADA, Natalia, 2005.
- La fase 1 parte en el horror e impacto psicológico del primer minuto luego del evento, donde ocurre la reacción inmediata, surgen el pánico y la organización precaria de las primeras 3 horas y se podría esperar que llegue algún auxilio externo en ese lapso, pese a que lo normal es que no llegue nada. Por eso es importante tener elementos de auxilio y personas entrenadas en la comunidad, tal como ocurre con los sistemas de reacción rápida, en el municipio, JJVV o bomberos. Los bomberos locales, si es que a su vez salvaron su equipamiento, son la opción más rápida en la acción inmediata.

- Fase transitoria, extendiendo la misma fase 1. Viene la reacción en los primeros días hasta semanas post desastre, donde se implementan los sistemas transitorios de urgencia. Hay gente sin casa en los cerros, en la costa, en los pueblos, familias con la casa inhabitable, durmiendo en la calle, que necesitan cubrir muebles, alimentos, juntarse, cobijarse aunque sea mínimamente. Es muy común en esta etapa que se presente una crisis de agua potable, en los sistemas sanitarios y carencia de energía.

Esta fase es la de emergencia inmediata y tránsito, la fase de cubrir lo más rápido que se pue- 
da las necesidades urgentes. Es en esta fase que se inserta este proyecto.

- La fase 2 corresponde a limpiar, iniciar el duelo, evaluar y planificar lo que se va a hacer para seguir viviendo y reconstruir definitivamente. Esta fase llega incluso al diseño y construcción de nuevos edificios y pueblos. Lo normal en las tragedias de este tipo en Chile es que esta fase no comienza hasta después del primer o segundo mes y dura normalmente entre uno y tres años.

Por lo anteriormente descrito hay que entender que para una catástrofe en el tiempo, las acciones $\mathrm{y}$ ayudas son del todo diferentes, tanto en urgencia como en intensidad.

El proyecto que se describe en este documento transcurre en fase 1 , primeras semanas, trabajando en lo más urgente dentro de lo que resulta viable. Son tantas las demandas que hay que elegir algo factible, y eso fue lo que determinó este proyecto.

Muchas veces es lo menos recomendable el aumentar en los lugares afectados la cantidad de gente que necesita comer, dormir y consumir agua, donde por la emergencia no hay alimentos, techo, ni líquidos para los propios damnificados. Por lo anterior, en muchos casos es bienvenido el poder enviar armados, preparados y listos para usar los sistemas

132 revista invi № 72 / Agosto 2011 / Volumen № 26: 129-151 de ayuda inmediata. Es más, resulta fundamental que los tiempos de la intervención inicial y armado sean cortos.

\section{LAS VIVIENDAS DE EMERGENCIA}

Este es un tema aparte, sobre el cual hay mucho que discutir y acordar. De hecho, el módulo emergencia fue el tema propuesto por el concurso Bienal de Arquitectura 20105. Según las bases debía ser un módulo habitable, desplegable, transportable, con un costo no mayor a $\$ 500.000$, que era el tope definido por la mediagua en marzo de 2010. Los resultados indican que no hay nada definitivo al respecto, sobre todo al entender la emergencia en su fase adecuada y no confundir con los procesos de reconstrucción.

En la primera semana post terremoto estaba comenzando a discutirse el asunto de las mediaguas como viviendas de emergencia. Se sabía que esa sería la única opción probable para la sustitución de cobijo post terremoto. Las críticas profundas a la mediagua ${ }^{6}$ como vivienda de emergencia volvieron a hacerse presentes: las unidades se entregan sin sanidad, sin energía, sin agua, sin aislación térmica y con una aplicación supuestamente transitoria, que terminaba, como ha sido históricamente, convirtiéndose en definitiva, determinando así un es- 
tándar de pobreza posterior. A pesar de todo esto, las mediaguas seguían siendo la única propuesta viable y experimentada para estos casos. Sin embargo, había otro tema a superar en la realidad: la demanda de mediaguas superaba totalmente la capacidad de producción en el corto plazo, vale decir en fase 1, y la velocidad necesaria de cobertura en la urgencia no estaba resuelta. Por ello, el equipo ARQ-X del Departamento de Arquitectura de la UTFSM, determinó que una cobertura rápida, plegable y desplegable, a bajo costo, podía entregar una solución transitoria a la intemperie mejor y de menor costo que las cualidades de una carpa del mismo tamaño. Y más rápido que cualquier otra solución, como en el caso del mediaguas clásicas.

La idea de aplicar nuestro conocimiento en geodésicas a las coberturas rápidas y livianas de emergencia había comenzado en el proceso de investigación en la Unidad de Arquitectura extrema a partir del terremoto en Haití, el 12 de enero de 2010.

En una investigación en docencia de la UTFSM, sobre Outdoor Learning con una publicación $\mathrm{ISI}^{7}$, se habían desarrollado en taller de primer año de 2006 a 2009 una serie de ejercicios 1:1 en terreno, aplicando metodologías de resolución de problemas como parece en publicaciones del MECE-

7 SERRANO Rodríguez, Pedro y GONZÁLEZ Böhme, Luis Felipe, 2009.
SUP USA0604 ${ }^{\circ}$, a aprender haciendo o hands on learning.

En el ejercicio de primer año 2009, los 60 estudiantes habían levantado un taller de producción en serie y construido 8 estructuras geodésicas metálicas de 5 metros de diámetro, perfectamente gemelas, que se probaron en el Parque Interuniversitario de Valparaíso. Se pensó en enero de 2010, que el resultado del taller 2009 entregaba las estructuras básicas para armar una entrega solidaria en Haití. Luego de desarrollar y modelar las coberturas en PVC anti UV termosellado, el proyecto Haití cambió de giro cuando se produce en Chile, poco más de un mes más tarde, el terremoto $8,8^{\circ} \mathrm{R}$.

\section{La investigación previa}

Las estructuras geodésicas han estado desde siempre en la naturaleza, a nivel microscópico los fullerenos ${ }^{9}$ están hoy en todos los avances de la nanotecnología y nuevos materiales. Su nombre proviene de los trabajos de Westminster Fuller ${ }^{10}$, quien desarrolló para la arquitectura extensos y ya famosos estudios que le permitieron construir y proponer cubiertas geodésicas como soluciones de arquitectura estructural para grandes luces, resistentes y con poco peso material.

\footnotetext{
wWw.romboarq.blogspot.com

GONZÁLEZ Viñas, Wenceslao; MANCINI, Héctor, 2003, p. 206.

EDMONDSON, Amy C.
} 
El año 2005, en el Departamento de Arquitectura UTFSM, luego de varias experiencias con geodésicas de distintos tipos y grados, realizadas con nodos especiales y aristas clasificadas, se tomó un fullereno clásico de carbono 60 y se desarrolló una construcción innovadora, ya no por nodos y barras, sino que por caras hexagonales y pentagonales previamente consolidadas. La experiencia tenía dos antecedentes en la Escuela de Arquitectura de la Universidad de Talca ${ }^{11}$, con talleres realizados por el autor. Estas pruebas, también de outdoor learnig, bajo principios técnicos científicos, permitieron comprobar que para igual estructura de fullereno resultaba mucho más rápida la construcción por caras consolidadas de hexágonos y pentágonos piramidales que una construcción en terreno por nodos y barras.

Las caras consolidadas se unían por aristas de un modo rápido, a diferencia de la unión por nodos, que requiere instructivos y soportes más complejos.

A partir de estas constataciones en el proceso de investigación tecnológica, se continuó desarrollando la metodología de construcción por caras geométricas regulares previamente consolidadas y uniones por aristas, lo que duplica la sección de cada arista en el conjunto, mejorando esto la resistencia estructural usando aristas de menor sección.

11 QUEZADA, Wenceslao. 2009
La última prueba de producción se hizo en el proceso de taller del segundo semestre de 2009. Se propuso al equipo de primer año desarrollar un sistema semi-industrial en serie para la producción de un diseño en metal para geodésicas por piezas consolidadas de $5 \mathrm{~m}$. de diámetro. Tomado este modelo de la propuesta de ARQ-X, las geodésicas metálicas fueron exitosamente producidas y montadas en el campo de pruebas del Parque Quebrada Verde. Estas estructuras, todas iguales, con partes intercambiables y repuestos homogéneos, serían durante el primer semestre de 2010 cubiertas con tela especial tras un ejercicio, también de producción en serie, de sellado de telas aplicando modelación computacional. En enero de 2010 ocurrió el terremoto en Haití y se inició el proceso de preparación de módulos de ayuda transportables en los Hércules C130 de la FACH. Toda una modelación en taller que no se concretó finalmente, puesto que ocurrió el terremoto 27/2 en Chile.

\section{La propuesta de acción}

Durante la primera semana de marzo de 2010 el equipo ARQ-X planificó un proceso rápido de adaptación productiva del modelo de $5 \mathrm{~m}$. de diámetro para la emergencia nacional.

- Se tomó la decisión de adaptar rápidamente las 8 estructuras geodésicas que estaban aún en el

ARTíCULO: Geodésicos post terremoto. Investigación aplicada en la emergencia /

Pedro Serrano Rodríguez 
parque, pintarlas y mantenerlas, usando esta vez el patio central de la Universidad, con un equipo de voluntarios convocados por la Federación de Estudiantes UTFSM.

- El arquitecto de ARQ-X, Alejandro Véliz, desarrolló en software Rhino ${ }^{12}$ la modelación óptima para lograr la cobertura termosellada en tarpaulin $\AA^{13}$, un tipo de PVC usado en carpas de camping, que se seleccionó por ser accesible en Chile, resistente al UV, barato, firme y ligero.

- Tomando parte de la experiencia adquirida en la producción seriada y exitosa de 2009, se decidió, esa primera semana post terremoto, establecer un proceso productivo seriado, guiado por estudiantes, para construir geodésicas gemelas a las metálicas, esta vez con tubos de Poli Vinil Cloruro, para conducción de cables eléctricos, tipo Conduit ${ }^{14}$ de $25 \mathrm{~mm}$. color naranja, un material accesible en todas las ferreterías de Chile, fácil de trabajar con herramientas simples de corte y de calor.

- La organización de la producción se hizo con estudiantes voluntarios de $2^{\circ}$ año con la experiencia de 2009, se utilizó todo el espacio disponible en los patios UTFSM y el Departa-

12 RINOCEROS $®$, Nurbs modeling for Windows, Programa de modelación para diseño y arquitectura, http://www.rhino3d.com/

13 http://la.hxtarp.com/ProductList.asp?gclid=CKqX4luEpKcCFa5 k7AodByJhEg

14 Catálogo Conduit, www.socomsa.cl mento para colocar las mesas de trabajo con matrices para el PVC y costuras de la cubierta.

- La gestión de la primera semana logró recoger financiamiento inicial de la propia UTFSM y de dos oferentes internacionales en una cuenta interna propia.

- Se hicieron matrices y muestras de corte y confección de cada pieza.

- Se diseña el lay out completo de un espacio productivo en talleres y patios durante las dos semanas antes del ingreso a clases.

- Se establece un sistema de diagnóstico en terreno, por corresponsalía de estudiantes que viven en las regiones afectadas.

- Ya en la primera semana post terremoto, se inicia la producción seriada de geodésicas para cobertura rápida con estructura plástica y cubierta de Tarpaulin®.

- El sellado y costura de las telas pre cortadas según modelación fue asumido la tercera semana como aporte por parte de industrias Wieneke, en Viña del Mar' ${ }^{15}$.

- En paralelo, y recogiendo resultados inmediatos del sistema productivo, se desarrollan planos,

15 Wieneke fabrica velámenes para barcos y yates, a la vez que fabrica carpas de camping, y posee las herramientas y tecnología para hacer las cubiertas 
cubicaciones y manual del sistema productivo y se publican en la web de la Universidad; el material es copiado en varias web solidarias al proyecto $^{16}$, de modo que las instrucciones para su producción en cualquier lugar del planeta fuese libre, factible y sin costo para nadie.

- A medida que se terminan las estructuras y cubiertas, los domos comienzan a repartirse en distintas zonas y comunidades del área afectada.

\section{La condiciones del programa}

La primera y más importante definición de uso es que estos Domos de Emergencia "son coberturas impermeables y sólidas, armadas sobre una estructura geodésica de 5 metros de diámetro, pensadas para la emergencia de no contar con cubierta para bodegas, comedores, enseres, reuniones, incluso para dormir en casos extremos. A diferencia de una carpa tienen estructura sólida, tienen 3 metros de alto, por lo tanto son un GRAN espacio cubierto, mucho más barato que las alternativas, al costo de producción final logrado de 260 USD con mano de obra voluntaria".

- "No son casas, no pretenden serlo y no sirven para ello". Esta advertencia va en las cubiertas y los manuales de armado.

16 PASTORELLI, Giuliano. 2010

136 revista invi № 72 / Agosto 2011 / Volumen № 26: 129-151
- Sirven de apoyo para el proceso post desastre inmediato y también luego, para los procesos de la fase 2

- Pertenecerán a la comunidad. Si el usuario asignado dejó de necesitarlo, lo puede pasar a otro vecino o guardar plegado en un lugar comunitario hasta que vuelva a ser necesario.

- Una vez terminada la emergencia las cubiertas geodésicas se desarman fácilmente, se empacan y se debieran guardar plegadas en la Junta de Vecinos, la municipalidad local, los bomberos o la Onemi, o en caso de no existir otra opción, se debieran devolver a la Universidad, todo listo para una nueva emergencia. No se regalan, ni a familias ni a personas; pertenecen en primera instancia a la comunidad.

- El diseño ha sido desarrollado por profesionales de Arquitectura de la Universidad Técnica Federico Santa María para su confección por grupos de voluntarios, una futura microempresa o talleres solidarios (ver manuales publicados, otras páginas) ${ }^{17}$.

- Las coberturas geodésicas sirven de apoyo para el proceso en el desastre inmediato y también de apoyo posterior para el proceso de transición en la recuperación de viviendas, puesto que cuando se tiene que demoler la casa dañada para construir la nueva, es necesario un

17 Instructivo $\mathrm{N}^{0} 1$ de 4.2010 
refugio de transición, que por supuesto no se convierta en definitivo.

- El modelo con todos sus planos quedó disponible para su réplica en otras escuelas de arquitectura o grupos voluntarios sin destino comercial.

- En el equipo ARQ-X se sabía además que si la universidad no proponía otra cosa, las clases normales se iniciarían en dos semanas más, luego de iniciado el taller productivo de cubiertas geodésicas, por lo que el personal voluntario tanto del Departamento como de la Federación de Estudiantes terminaría de presentarse bruscamente.

- De hecho, en las últimas producciones hubo que pagar la mano de obra, duplicándose el costo de cada unidad (600 USD).

- Académicamente lo importante era que el terremoto, más allá de la tragedia, había dado la oportunidad de probar en la realidad, en terreno y con condiciones de tiempo apremiantes, que todo lo desarrollado durante años de investigación por ARQ-X en cuanto a geodésicos tenía una aplicación clara, necesaria, muy concreta y viable.

- También fue condición imperativa del programa el compartir abiertamente el conocimiento sin cortapisas; de este modo se colocaron todas las bases, fotografías, plantas, matrices y pla- nos a disposición de la comunidad nacional e internacional, por medio de la varias páginas web que solidarizaron y en documentos en papel que se entregaron a personas o grupos que los solicitaron. De hecho, se han recibido por internet decenas de solicitudes, cartas de apoyo y preguntas específicas desde todo Chile y otros países.

\section{La organización productiva}

La organización tuvo un equipo central que coordinó todos los aspectos del proceso, desde el diseño de partes y piezas, lo financiero, lo administrativo, los procesos de modelación computacional y cálculo, la distribución en terreno, hasta el desarrollo tecnológico del proceso productivo. Este equipo fue constituido por los profesionales de la Unidad de Arquitectura Extrema, ARQ-X, del Departamento de Arquitectura UTFSM, con asiento en la Casa Central de la UTFSM en Valparaíso. Se dispuso de los espacios de los talleres lectivos, laboratorio de materiales, equipado con cortadora CNC y cortadora Laser, acceso al laboratorio de ciencia de materiales y al equipamiento del laboratorio de bioclimática (Cuadro 1).

En el equipo central se desarrollaron tres líneas principales de acción: 
- La primera fue la encargada de la gestión productiva, que desarrolló los manuales de producción con el proceso web, manejó los equipos de voluntarios, coordinó a distancia el montaje en terreno, capacitó y transfirió experiencia a otros productores.

- La segunda correspondió a la gestión financiera, búsqueda de financiamientos, compras de herramientas, materiales, equipamiento y rendiciones a la cuenta UTFSM del proyecto. Esta sección se encargaba además de los despachos y fletes a localidades.

- La tercera fue la gestión social en terreno, que partió con nuestros propios estudiantes contactados en los lugares afectados y luego siguió con misiones de estudiantes que viajaban a las localidades detectadas por solicitudes telefónicas e internet. Estos mismos equipos luego coordinaban las acciones en terreno, consiguiendo además voluntarios locales e incluso aportes de dinero, alojamiento, transporte y alimentación de los equipos en terreno. Una buena parte de las veces había que hacer trabajos de terreno como nivelaciones o plataformas.

En la escuela de Arquitectura de la UTFSM se montaron mesas y matrices de corte, maquinarias, mesones de producción, espacios de almacenamiento de insumos, embalaje y despacho de geodésicos terminados (Cuadro 2).
Cada geodésico embalado y enviado contaba con información básica estampada en la tela de cubierta y llevaba en un sobre impermeable un manual completo para el armado.

La mayor parte de las veces un equipo, o incluso un solo voluntario entrenado, viajaba y acompañaba el proceso local de construcción. Sin embargo, hubo casos en que la comunidad local, tan solo con el manual, pudo levantar sus geodésicos de emergencia

Los tiempos de montaje, dependiendo de las circunstancias locales, estuvieron entre los $30 \mathrm{minu}-$ tos y las 2 horas por unidad terminada.

\section{Diseño, planos y manuales}

El diseño original de la estructura geodésica del módulo usado corresponde al autor. Ha sido desarrollado durante los procesos de investigación previa, siendo primero prototipado 1:1 en PVC de 1/2 "luego escalado a piezas metálicas de acero de $1 / 2$ " y finalmente escalado a piezas de PVC de $32 \mathrm{~mm}$. Esto se puede ver en instructivo 4, figura 2.

La cobertura de Tarpaulín ${ }^{\circledR}$ tuvo dos modelaciones, la primera de una esfera en proyección plana y la segunda en gajos. La segunda resultaba más fácil para productores en terreno sin acceso a computadores y máquinas de corte. 
Un equipo de profesores se encargó de las labores de modelación computacional y también de organizar los equipos de voluntarios. Al mismo tiempo, otro equipo se encargó del diseño y edición de los manuales de trabajo, los mismos que se subieron a la web para acceso gratuito de personas e instituciones. Las tres primeras semanas hubo 1800 visitas a los planos e instructivos de producción y montaje. De hecho, el buscador Google dio acceso directo y prioritario a quienes buscan en "Domos" o "Geodésicos de emergencia".

La idea principal del proceso de diseño de instructivos y manuales estaba en la posibilidad urgente de que otros grupos, entidades, escuelas de arquitectura, ONGs, pudiesen replicar con facilidad el sistema y así levantar con urgencia un poder productivo más abierto y ojalá localizado.

La publicación abierta de los manuales provocó gran interés de diversos grupos e instituciones. Algunas, como la Escuela de Arquitectura de la Usach, iniciaron e incluso perfeccionaron la reproducción del modelo.

A mitad del proceso se logró un convenio con la fábrica Wieneke, de Viña del Mar, especializada en la producción de carpas, para la confección de las cubiertas en máquinas industriales a partir de los cortes precisos que aparecen en los manuales, hechos por los voluntarios en la UTFSM usando las matrices digitales.
Wieneke llegó a confeccionar 13 cubiertas con su logotipo.

Se realizaron cuatro instructivos para la producción y el montaje. En cada instructivo se definieron las herramientas (Figura 1), los materiales, sus características y las dimensiones exactas para cortes, dobleces y uniones. Todo el material fue analizado y actualizado en la fase productiva, de modo que los instructivos que hoy es posible bajar de la red están plenamente probados, tanto en la fabricación como en el montaje en terreno.

Especial cuidado se puso en el diseño de los mismos, de modo que fuesen entendibles y aplicables por cualquier persona con habilidades para su producción con materiales simples y herramientas domésticas.

La publicación provocó una alta demanda de indicaciones, consultas y pedidos de personas de todo el país, e incluso desde el extranjero, demanda que se mantuvo todo el año 2010. La demanda de información y complementos se ha mantenido a pesar de que la fabricación en la UTFSM se detuvo finalmente en el mes de mayo de 2010.

\section{Gestión}

Los terremotos no avisan su ocurrencia, por lo tanto la capacidad de los equipos profesionales de reaccionar oportunamente y con la velocidad ne- 


\section{CUADRO 1}

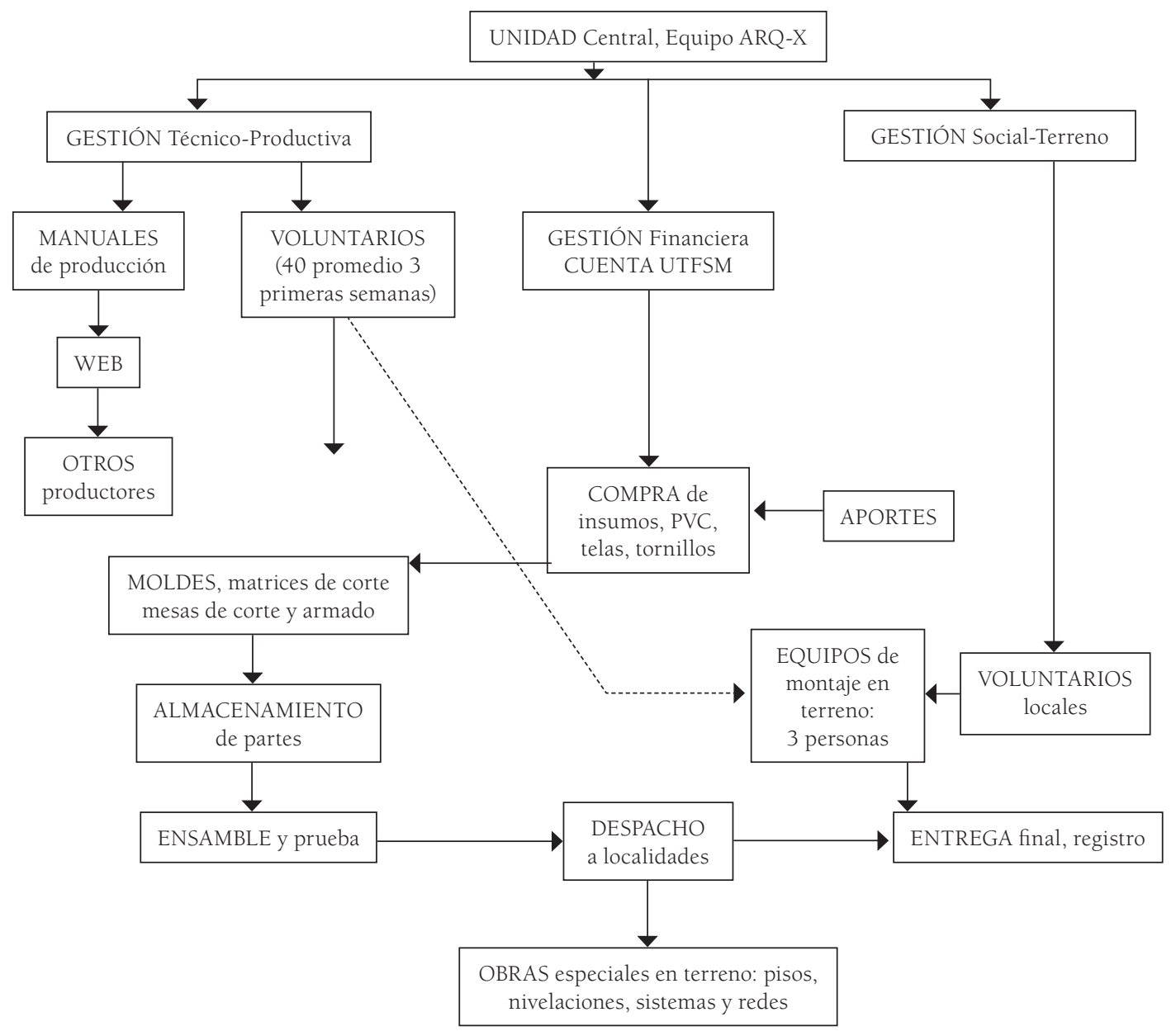

FUENTE: Elaborado por el autor 
CUADRO 2. PROCESO PRODUCTIVO

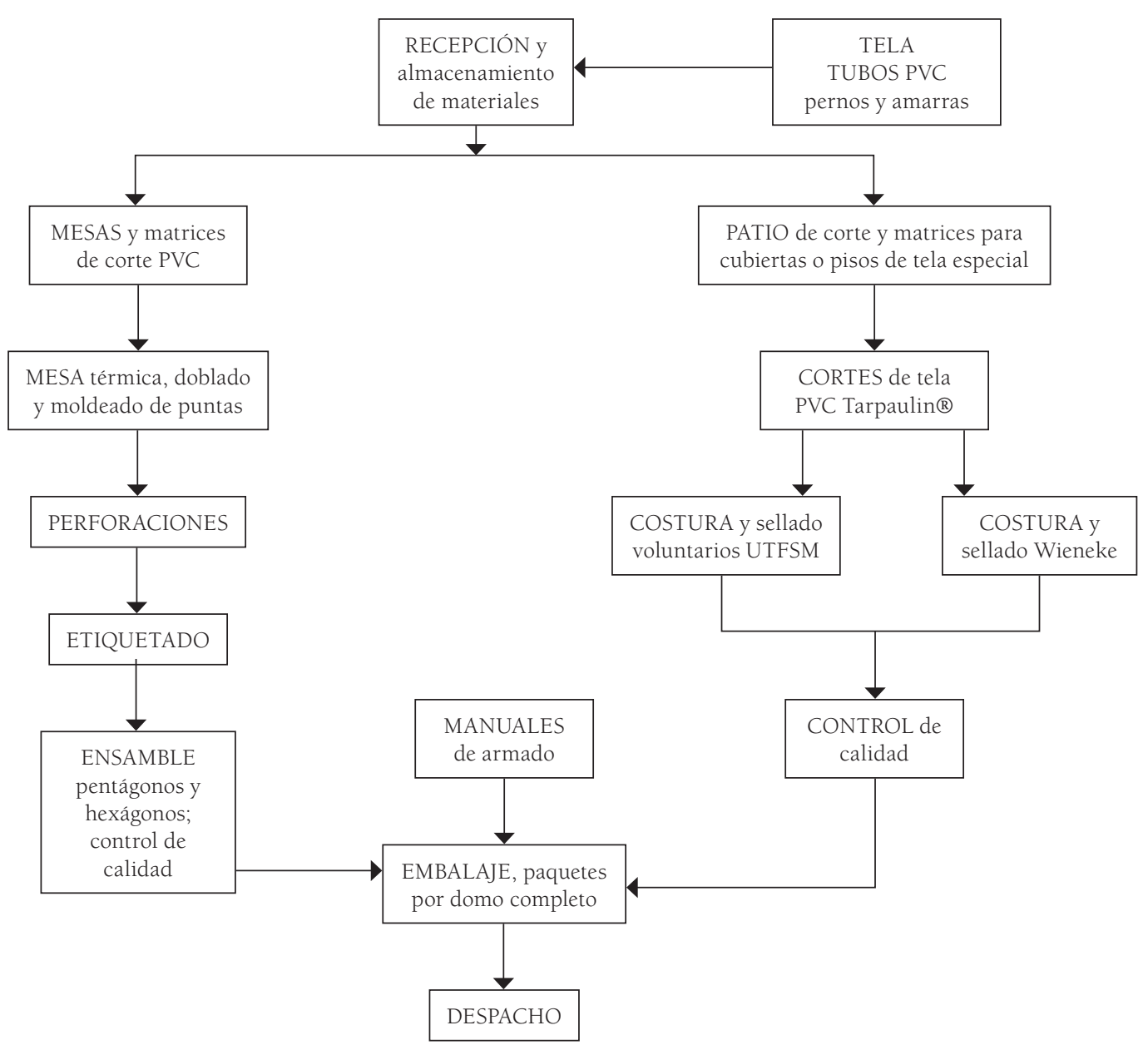

FUENTE: Elaborado por el autor

ARTíCULO: Geodésicos post terremoto. Investigación aplicada en la emergencia / Pedro Serrano Rodríguez revista invi № 72 / Agosto 2011 / Volumen № 26: 129-151 141

6410 Revista INVI 72 Interior.indb 141

23-08-2011 16:35:16 
cesaria se pondrá siempre a prueba en casos como este. De hecho, por dar un ejemplo local, el asunto de la ayuda para la emergencia no fue un tema prioritario en la primera reunión post terremoto del Departamento de Arquitectura; aparentemente había una preocupación marcada por la continuidad del cotidiano académico, hubo preocupación por ayudar técnicamente a la evaluación de daños, lo que se hizo en un comienzo con personal damnificado de la propia universidad, pero cuando ARQ-X plantea el tema de construcciones específicas y rápidas, como es el caso de este proyecto de geodésicas, hubo más oposición, dudas y cuestionamientos que voluntad de acción. En situaciones como esta, en que había una real emergencia y una propuesta concreta, hubo que tomar una decisión dividida, actuar sin consensuar. ARQ-X, con un grupo de profesores también voluntarios, asume la gestión de la propuesta, ante la inminencia de la realidad y la imposibilidad de levantar un debate académico sobre el caso en tiempos que por la emergencia no estaban disponibles.

Inmediatamente después de la reunión se concertó una reunión con la Rectoría y la Vicerrectoría Económica y Administrativa de la UTFSM, logrando rápidamente un aporte de $\$ 2.000 .000$ y la apertura de una cuenta interna a nombre del autor. La Universidad conocía los proyectos de módulos y distintos geodésicos y confiaba en la capacidad productiva de ARQ-X, y entendió además que en la emergencia era mejor actuar, lo que prestigiaba de todas formas a la Universidad.

Además a partir de esto, tomada la decisión en un día y con financiamiento inicial de la Universidad, se logró ese mismo día organizar los equipos de trabajo, tal como aparece en el cuadro 1. Rápidamente se recuperaron, repararon y pintaron con antióxido los geodésicos del Parque Quebrada Verde, mientras simultáneamente se llamaba a los voluntarios de arquitectura y de la Federación de Estudiantes de la Casa Central.

Simultáneamente se desarrollaron los patrones digitales para las cubiertas, se cotizó la membrana de cobertura y se encargaron los tres primeros rollos de material de cubierta facturando a la UTFSM.

Ya dentro de la primera semana estaban funcionando correctamente los patrones de corte y las matrices de tuberías de PVC, con una acumulación básica de materiales listos para corte. Los estudiantes voluntarios del Departamento con experiencias previas con el producto, capacitaron técnicamente a los voluntarios de la Federación Central de Estudiantes de la UTFSM. Se organizaron turnos y gestión de herramientas y manutención sobre la marcha.

Como un proceso de control de calidad y un estímulo motivacional visual para toda la comunidad, se levantaron en el patio principal las 8 geodésicas metálicas existentes, y ya el segundo lunes se hizo una inauguración de los primeros produc- 
FIGURA 1. MANUAL. PORTADA INSTRUCTIVO 1

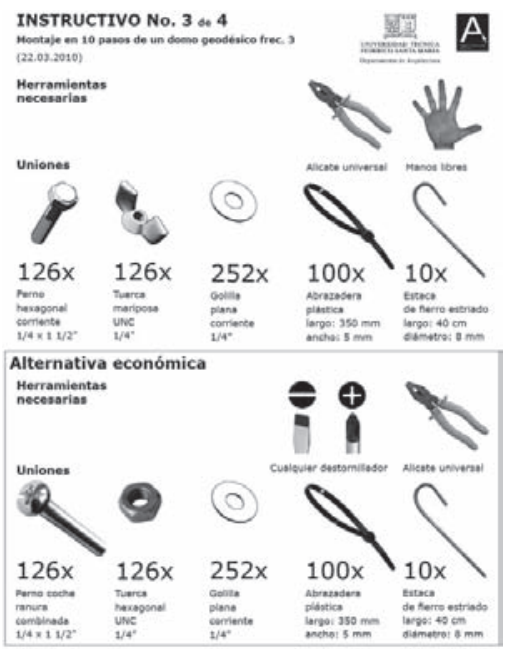

FIGURA 3. PLANTILLA ESFÉRICA

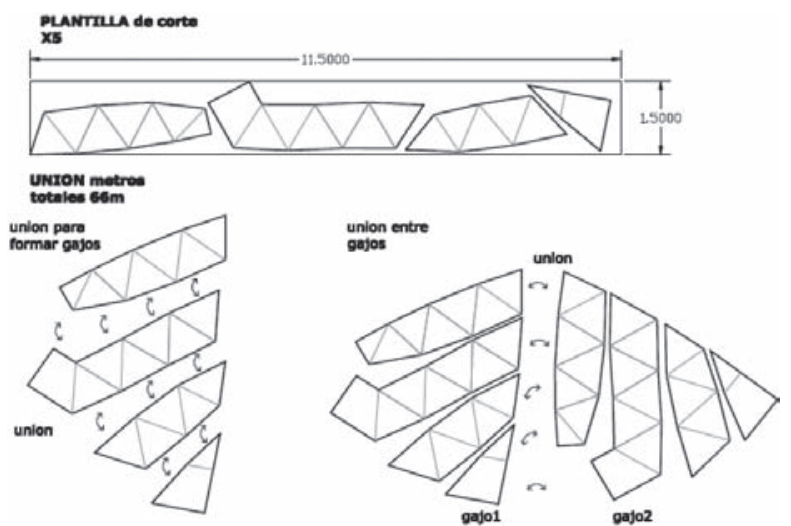

ARTíCULO: Geodésicos post terremoto. Investigación aplicada en la emergencia / Pedro Serrano Rodríguez
FIGURA 2. MANUAL. GAJOS DE CUBIERTA

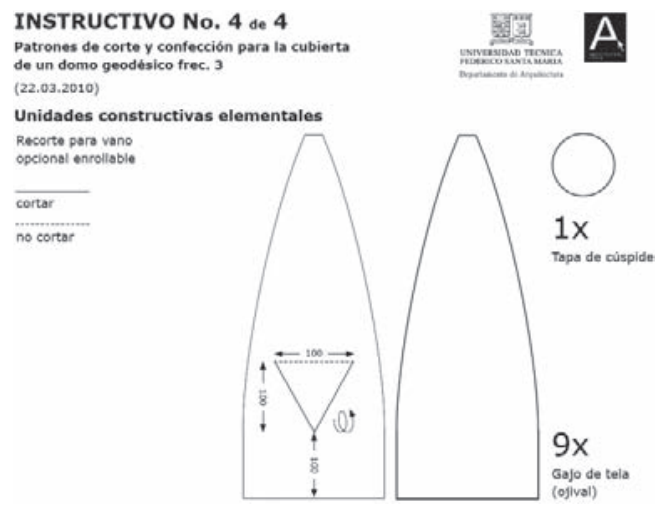

FIGURA 4. MANUAL. SISTEMA DE ARMADO

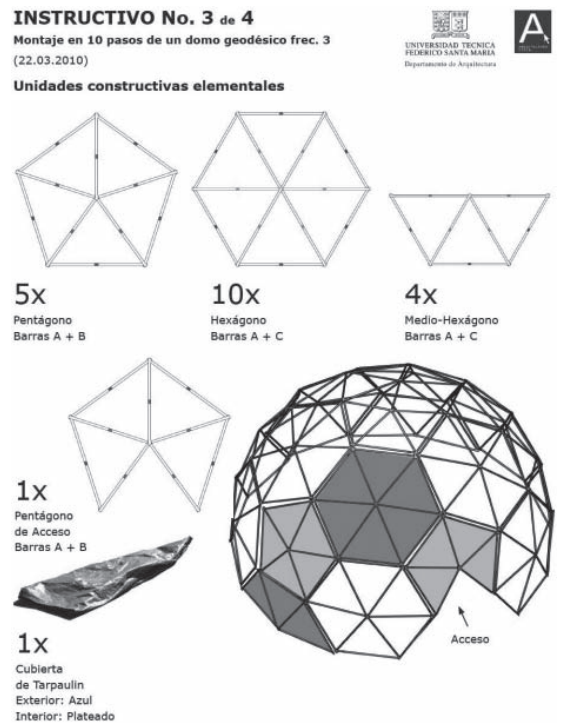

revista invi № 72 / Agosto 2011 / Volumen № 26: 129-151 143 
tos en despacho con asistencia de las autoridades universitarias.

Se logró conectar dentro de la semana la posibilidad de recibir aportes en dólares y euros en la cuenta, con bastantes problemas administrativos, recibiéndose al final aportes en pesos y un aporte en euros desde personas amigas en Europa ${ }^{18}$. Los aportes de institución a institución no fue posible consolidarlos.

También existió en principio una auspiciosa oferta inicial de un importante grupo económico nacional, la que fue cancelada con la realización de la Teletón, ya que los oferentes decidieron que su aporte tenía más impacto mediático en este evento, lo cual es evidente.

La parte más complicada de la gestión, que podemos nombrar como gestión de la ayuda en plena emergencia, fue definir y decidir las entregas a contactos o solicitantes desde las localidades afectadas. Algunas veces el contacto fue el propio municipio, otras veces la intendencia, algún Seremi, el encargado local de educación, la oficina de Juan Fernández en Valparaíso, alguna junta de vecinos contactada por los estudiantes, asociaciones de artesanos o postas de salud. Por razones obvias, no se atendieron solicitudes individuales, que fueron muchas. A solicitantes individuales se les sugirió actuar con alguna organización local y a otros, por

18 Celestino Ruivo R, Universidad de Algarve, Portugal su propia consulta y motivación, recibieron copias de los manuales con los sistemas productivos.

Un esfuerzo notable de los profesores y ayudantes que participaron voluntariamente en el proceso fue el desarrollar un manual bien diseñado, con todas las medidas y procedimientos de producción ordenados educativamente, como para que cualquier persona con habilidades manuales convencionales y herramientas domésticas simples, pudiese enfrentar la autoconstrucción del modelo, asunto que tuvo réplicas incluso después de la emergencia, con grupos de teatro que hicieron geodésicas itinerantes para su actividad artística en distintas localidades.

\section{Impactos, conclusiones}

Los geodésicos de emergencia UTFSM llegaron desde Isla Juan Fernández hasta Talcahuano, siguiendo una línea de contactos y diagnósticos cercanos y de solicitudes desde la zona afectada que llegaban por teléfono o internet.

Todo este proceso de realizó dentro de los primeros 3 meses de la emergencia, cumpliéndose con lo esperado para la etapa de transición en la primera fase post catástrofe.

En general se entendió el uso de los geodésicos de emergencia diseñados como cubierta transitoria, no siendo utilizados como vivienda. 
Por presupuesto y tiempo, los estudiantes voluntarios entraron a clases y no se cubrió completamente la demanda de solicitudes de todo tipo llegadas a la unidad de coordinación. Pasados los primeros tres meses se entendía que correspondía el inicio de la fase 2 , de reconstrucción.

Las geodésicas de emergencia se usaron en la realidad como:

- Salas de clase de escuelitas uni-docentes en comunas alejadas que habían perdido totalmente sus edificios.

- Sala de clases en la U. de Talca, taller de transición y producción de Arquitectura de la Usach.

- Centros comunitarios en varias localidades.

- Salas de atención transitoria en postas rurales.

- Salas de reunión de personas.

- Bodegas de sistemas de ayuda (salud, alimentación).

- Bodegas de tránsito de enseres de viviendas.

En algunos proyectos, en uso comunitario, se construyeron pisos sobre pilotes de madera; en otros casos, como el de las escuelitas uni-docentes, tuvieron pisos del mismo Tarpaulin® de cubierta, que dicho sea de paso es la tela más común usada para piso de carpas de camping.

ARTÍCULO: Geodésicos post terremoto. Investigación aplicada en la emergencia /

Pedro Serrano Rodríguez
- Cuatro grupos, incluido uno de arquitectura de la Usach, iniciaron producciones propias.

- Se recibieron docenas de consultas de grupos, instituciones y personas que habían bajado los manuales de los sitios web, tanto en Chile como en el extranjero.

- Localidades alcanzadas por el grupo UTFSM:

San Vicente de Tagua Tagua,

Isla Juan Fernández

Forel

Pichamán

Macal

Valparaíso

Matanzas

Rancagua

Talca

Quilpué

Constitución

Concepción

Mataquito

Aún resta por saber qué ocurrirá con las coberturas geodésicas a un año del proceso (marzo-abril de 2011).

Está pendiente una evaluación participativa de los resultados en terreno, y el registro web aún no se cierra, puesto que a marzo de 2011 aún llegan consultas y solicitudes a la web. ${ }^{19}$

19 INSTRUCTIVOS para fabricar domos geodésicos de emergencia. 2010

revista invi № 72 / Agosto 2011 / Volumen № 26: 129-151 145 
El proceso descrito fue transitorio para la Universidad. El equipo de gestión fue absorbido luego del segundo mes por la actividad académica convencional; la página web y las solicitudes de información continúan activas hasta el día de hoy. Una conclusión importante es que se cumplió con la transitoriedad de una propuesta en verdad transitoria. A partir de esto se alimenta una reflexión importante acerca de cuál podría ser el papel de una universidad técnica y una escuela de arquitectura en los casos de ocurrencia de catástrofes de estas características, que por supuesto se sabe que seguirán ocurriendo. Resulta curioso verificar que para las autoridades la inminencia de una repetición de la catástrofe no genera actitudes permanentes. Es probable que una nueva catástrofe vuelva a abrir en las universidades una ventana ya olvidada. La sistematización de esta experiencia dentro del carácter netamente científico de la misma no es lo mismo que sistematizar el complejo mundo socio-organizacional que se da en los sitios de la catástrofe. De hecho, a marzo de 2011 aún hay dudas sobre las reacciones oficiales durante y después de la catástrofe.

En lo nacional, el aporte de la UTFSM pasó con muy bajo perfil, confrontado con la reacción del Estado y la magnitud de la catástrofe. El trabajo logrado fue una comprobación en terreno de algunas hipótesis planteadas en la investigación de años respecto de la aplicabilidad, la velocidad de montaje, la posibilidad de producción, el bajo costo y la usabilidad planteada en los momentos de tránsito. Esto con satisfacción lo consideramos exitoso, sobre todo por la oportunidad de corregir errores y supuestos teóricos en la práctica real.

La Universidad Técnica Federico Santa María es, además, una universidad de reconocido prestigio científico-tecnológico, por lo tanto el Departamento de Arquitectura UTFSM, rodeado de laboratorios complejos y carreras de ingeniería, tiende por su propio contexto a desarrollar una arquitectura donde la ciencia, la tecnología y la sustentabilidad están claramente incorporadas en la formación e investigación que desarrolla. De hecho, la presentación de este documento obedece a la culminación de un proceso de investigación científico, tecnológico y de investigación en docencia que fue finalmente probado en la práctica de un contexto real.

\section{Equipo participante:}

Pedro Serrano Rodríguez (Jefe de Proyecto y Coordinación general); Alejandro Véliz (Modelación y diseño Computacional); Fernando Hammersley, Luis Felipe González, Raúl Solís, Michele Berhó, Marcela Soto; Esther Engelmann (diagramación de manuales); Susan Carrasco, Valentina Rozas (coordinación de voluntarios e implementación en terreno); Lu Zhang, Esteban Uribe, Alejandro Muñoz, Pablo Barría, Juan Pablo Fernández. 


\section{Imágenes del proceso}

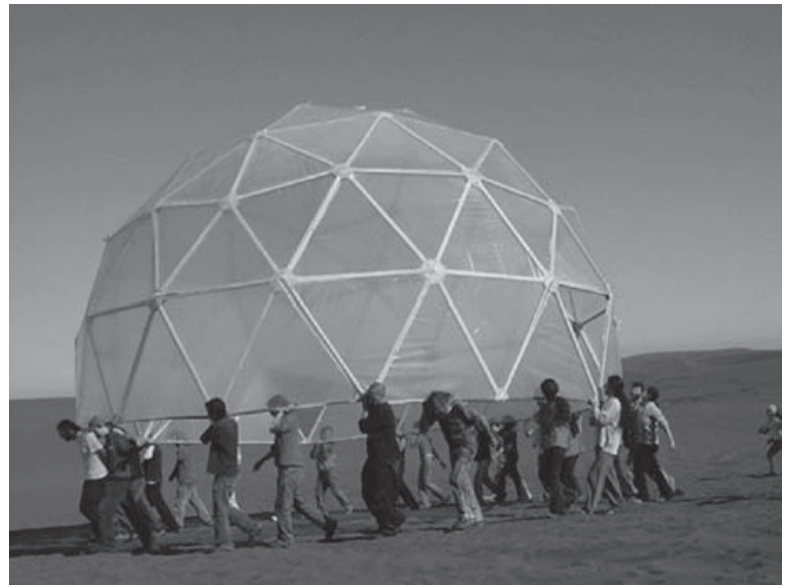

FOTO 1. Talca, Impacto cero Junquillar (2005).

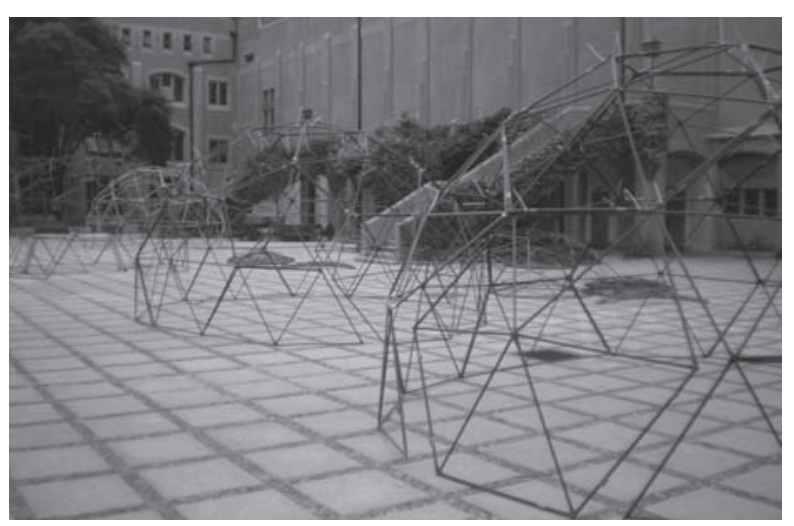

FOTO 3. taller geodésicas ler año

ARTíCULO: Geodésicos post terremoto. Investigación aplicada en la emergencia / Pedro Serrano Rodríguez

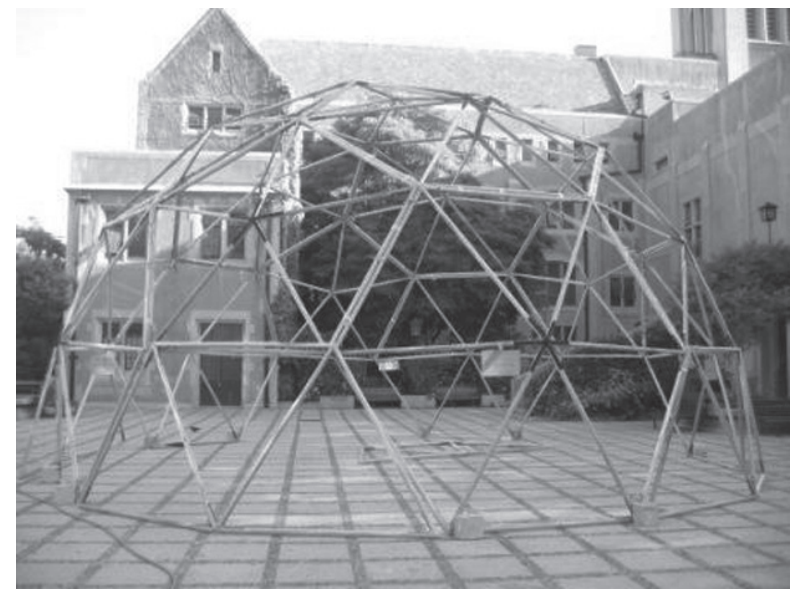

FOTO 2. UTFSM fullereno de C-60 (2006).

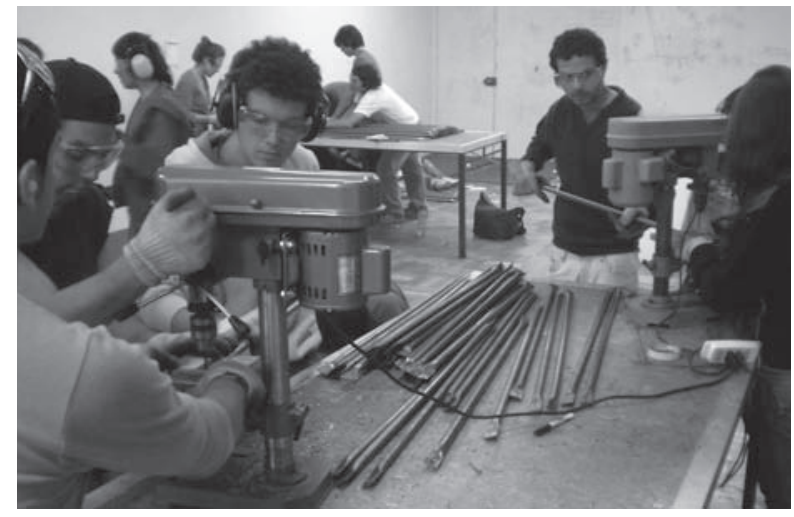

FOTO 4. Prototipo PVC

revista invi № 72 / Agosto 2011 / Volumen № 26: 129-151 147 


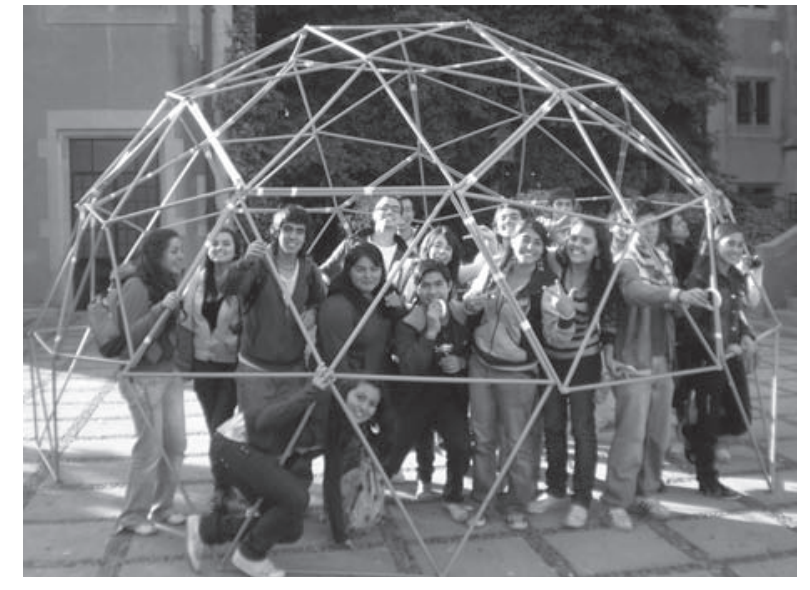

FOTO 5. voluntarios armando productos

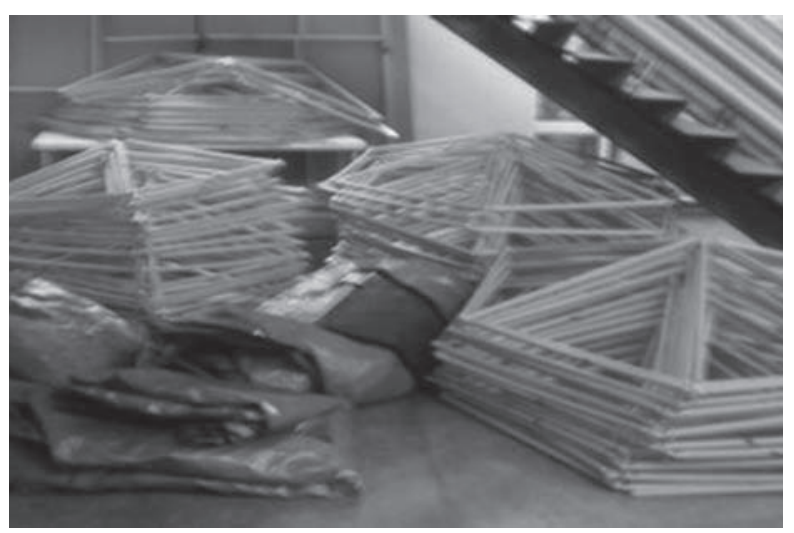

FOTO 7. paquetes de entrega

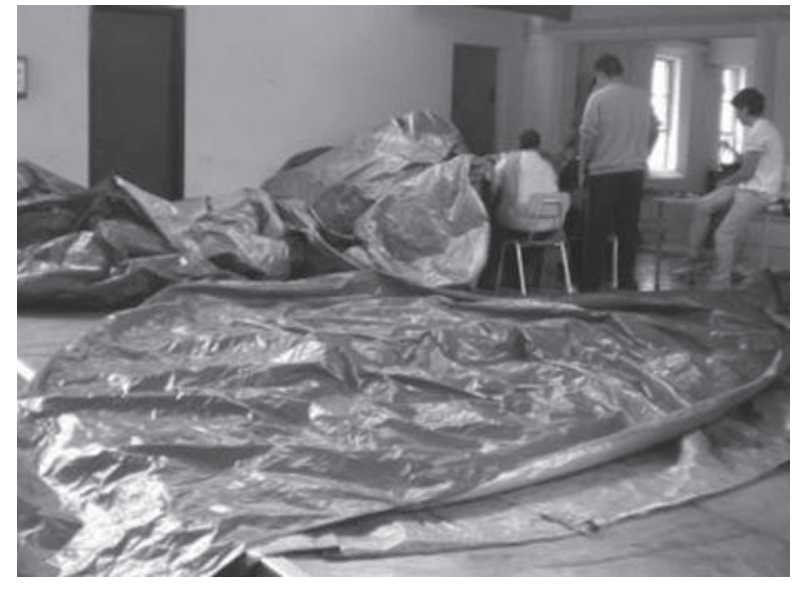

FOTO 6. Coberturas Tarpaulin

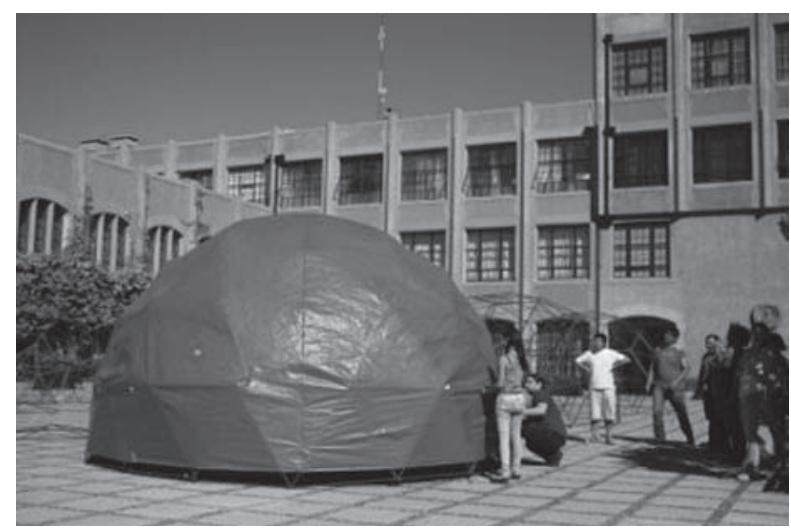

FOTO 8. pruebas de domos terminados

ARTíCULO: Geodésicos post terremoto. Investigación aplicada en la emergencia /
Pedro Serrano Rodríguez
148 revista invi № 72 / Agosto 2011 / Volumen № 26: 129-151
ARTICULL: Geodésicos post terremoto. Investigación aplicada en la emergencia /
Pedro Serrano Rodríguez 


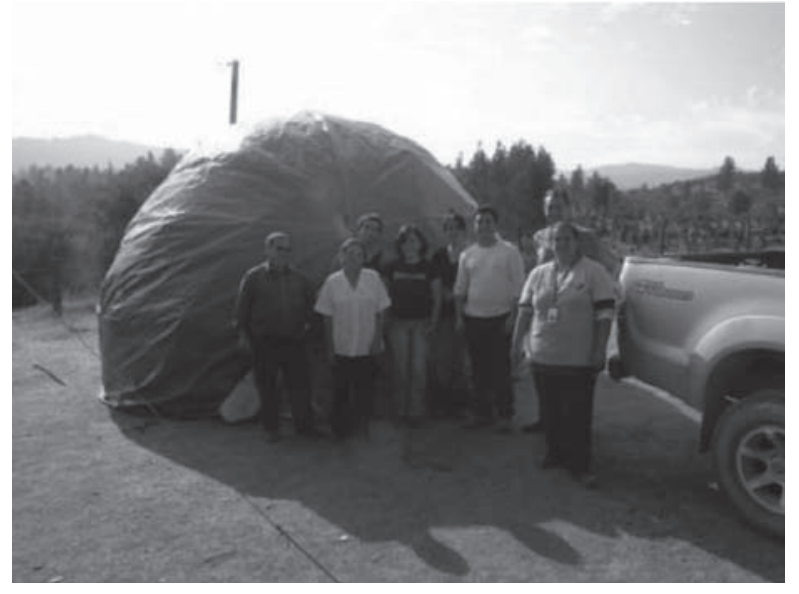

FOTO 9. Escuelita rural de Macal

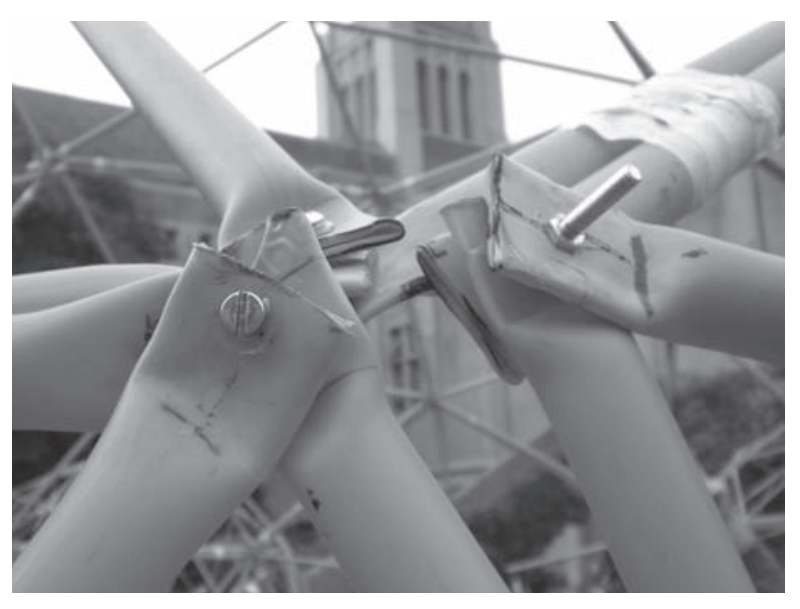

ARTíCULO: Geodésicos post terremoto. Investigación aplicada en la emergencia / Pedro Serrano Rodríguez

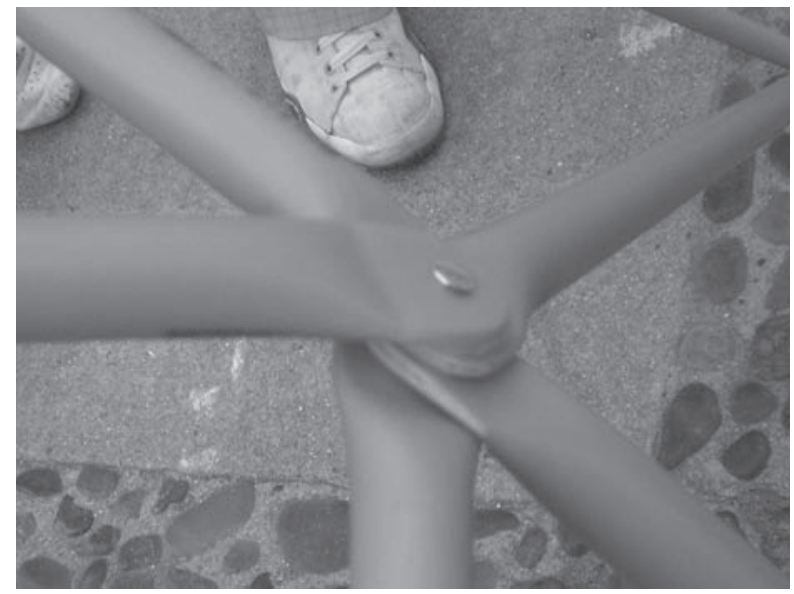

FOTO 10. Tipos de unión

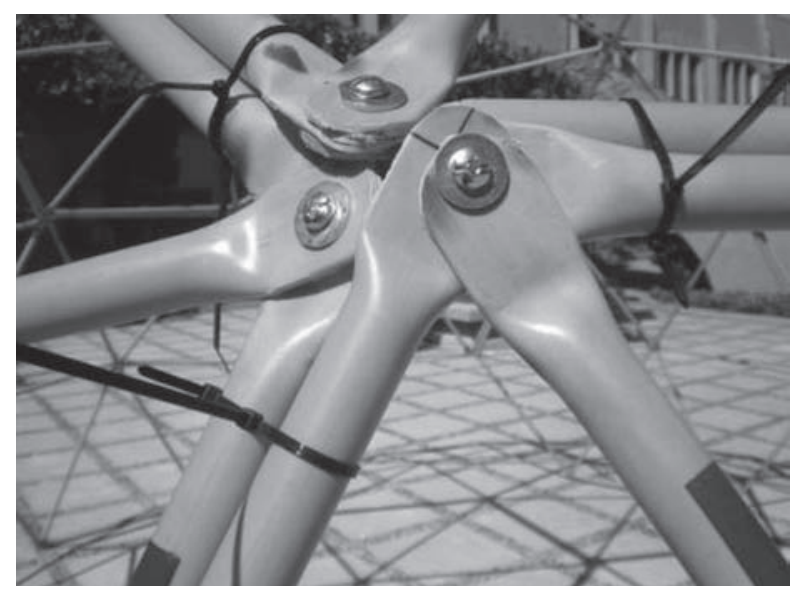

revista invi № 72 / Agosto 2011 / Volumen № 26: 129-151 149 


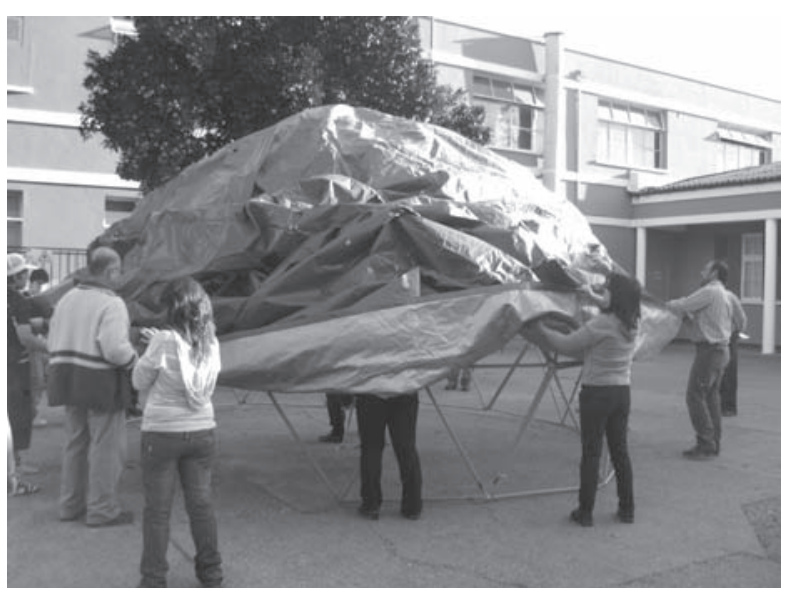

FOTO 11. montaje de cubierta San Vicente T.T.

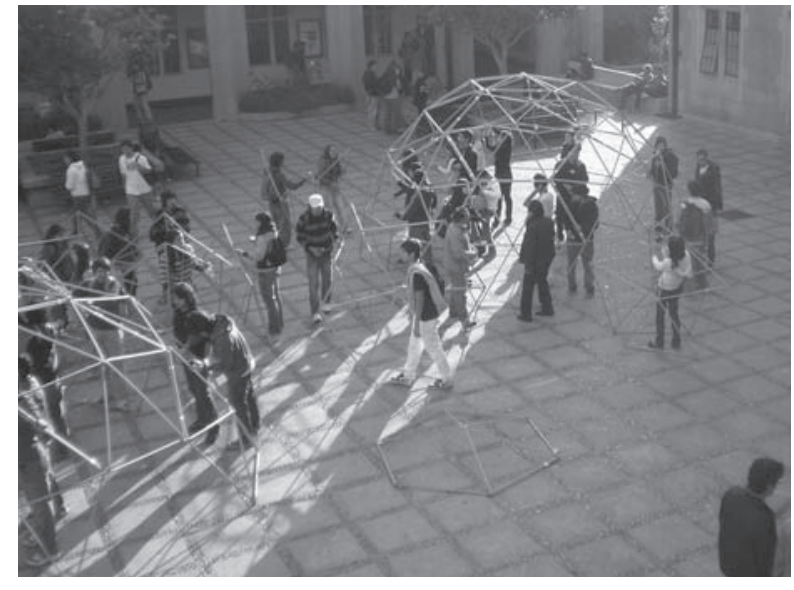

FOTO 12. patio central UTFSM
150 revista invi № 72 / Agosto 2011 / Volumen № 26: 129-151
ARTÍCULO: Geodésicos post terremoto. Investigación aplicada en la emergencia /

Pedro Serrano Rodríguez 


\section{Referencias bibliográficas}

AHUMADA, Natalia [et al] Vientos, terremotos, tsunamis y otras catástrofes naturales: historia y casos latinoamericanos. Buenos Aires, Biblos. 2005. 159 p. ISBN 950-786-498-9.

CÁMARA Chilena de la Construcción. Comisión Permanente de la Vivienda. Balance de la vivienda en Chile: análisis de la evolución sectorial, estimación de requerimientos habitacionales y proyecciones en el mediano plazo. Santiago, CChC. 2005. 109 p. ISBN 9568306021.

EDMONDSON, Amy C. A Fuller explanation: the synergetic geometry of R. Buckminster Fuller. Pueblo, Colorado, EmergentWorld. 2007. 339 p. ISBN 061518314X

GONZÁLEZ Viñas, Wenceslao; MANCINI, Héctor L. Ciencia de los materiales. Barcelona, Ariel. 2003. 228 p. ISBN 843448059X

INSTRUCTIVO No. 1 de 4 . Fabricación de barras en 5 pasos. Domo geodésico frecuencia 3. [En línea] Plataforma Arquitectura. Marzo 2010 [Fecha de consulta: marzo 2011]. Disponible en: http:// www.plataformaarquitectura.cl/wp-content/ uploads/2010/03/1269273451-1269238965-622-1-14-noti-doc.pdf

INSTRUCTIVOS para fabricar domos geodésicos de emergencia. [En línea] Departamento de Arquitectura, USM. Marzo 2010. [Fecha de consulta: marzo 2011]. Disponible en: http://www.arq.utfsm.cl/ instructivos-para-fabricar-domos-geodesicos-deemergencial
PASTORELLI, Giuliano. Construcción de un domo de emergencia: paso a paso. [En línea] Plataforma Arquitectura. Marzo 2010. Disponible en: http:// www.plataformaarquitectura.cl/2010/03/22/ construccion-de-un-domo-de-emergencia-pasoa-paso/

QUEZADA, Wenceslao. Geodésica de bambú. [En línea] Mediorural en el medio. Noviembre 2009. [Fecha de consulta: marzo 2011]. Disponible en: http://arrebol.org/mediorural/index. php?option=com_content $\&$ view $=$ article $\&$ id $=47$ : geodesica-bambu\&catid=1:noticias

QUEZADA, Wenceslao. Impacto CERO. [En línea] Mediorural en el medio. Noviembre 2009. [Fecha de consulta: marzo 2011]. Disponible en: http:// arrebol.org/mediorural/index.php?option =com content\&view = article $\&$ id $=50$ : i mpactocero\&catid $=1$ : noticias

SAN JUAN Guillén, César. Catástrofes y ayuda de emergencia: estrategias de evaluación, prevención y tratamiento. Barcelona, Icaria. 2001. 278 p. ISBN 8474265495.

SERRANO Rodríguez, Pedro; GONZÁLEZ Böhme, Luis Felipe. Exploring outdoor education and research in architecture. Open House International OHI. 34(1): 94-103. March 2009. ISSN: 01682601

UNIVERSIDAD de Chile, Departamento de Geofísica, Servicio Sismológico. Informe de sismo sensible. [Fecha de consulta: 23 diciembre 2010]. Disponible en: http://www.sismologia.cl

revista invi № 72 / Agosto 2011 / Volumen № 26: 129-151 151 